\title{
Patient perspectives on use of long-acting antipsychotics in bipolar disorder: focus on risperidone injection
}

This article was published in the following Dove Press journal:

Patient Preference and Adherence

17 August 2010

Number of times this article has been viewed

\section{Samalin \\ T Charpeaud \\ O Lorabi \\ PM Llorca}

Centre Hospitalier Universitaire, Clermont-Ferrand, France
Correspondence: $\mathrm{L}$ Samalin

Centre Médico-Psychologique B, Centre Hospitalier Universitaire, BP 69, 63003

Clermont-Ferrand Cedex I, France

Tel +33047375 2/25

Fax +33047375 2I 26

Email Isamalin@chu-clermontferrand.fr
Abstract: In the last few years, oral second-generation antipsychotics have demonstrated mood-stabilizing properties and are now widely used in the treatment of bipolar disorder. Unfortunately, treatment of this chronic and complex illness is hampered with poor adherence on the part of patients. Long-acting injectable formulations of second-generation antipsychotics could combine the effect of oral second-generation antipsychotics in patients with bipolar disorder and the benefits of depot formulation with the assurance of steady medication delivery and thereby improve adherence. In this context, the efficacy and tolerance of risperidone long-acting injection (RLAI) for maintenance treatment in patients with bipolar disorder is assessed. The relevant studies found RLAI to be effective in preventive treatment of manic but not depressive recurrences in bipolar patients, with good tolerance. RLAI appeared to be particularly suitable for patients with known poor adherence to treatment or severe bipolar disorder (such as patients who relapse frequently). Lastly, if RLAI, unlike the first-generation antipsychotics, does not induce depressive symptoms, the different studies do not enable us to consider its use in monotherapy in the preventive treatment of patients with depressive polarity. Long-acting second-generation antipsychotics in bipolar patients are therefore associated with long-term benefits, but their use in clinical practice needs to be improved.

Keywords: bipolar disorder, depot antipsychotics, long-acting risperidone injection, maintenance treatment, compliance

\section{Introduction}

Bipolar disorder is a chronic disease with a profound social and professional impact, and is particularly disabling, with a high suicide risk. ${ }^{1-3}$ Its periodic course and clinical heterogeneity require a complex psychosocial and pharmacologic approach, as well as control of compliance.

Treatment nonadherence or partial adherence is common in bipolar disorder. A review has reported nonadherence rates ranging from $20 \%$ to $66 \%$ across studies, with a median rate of $41 \% .{ }^{4}$ Nonadherence is the most important predictor of relapse and poor prognosis. Patients with bipolar disorder who fail to adhere to pharmacologic treatment have significantly higher rates of hospitalization than fully adherent patients. ${ }^{5}$ Considering that long-acting injectable antipsychotics improve adherence in schizophrenic patients, ${ }^{6}$ bipolar patients experiencing difficulties in maintaining good compliance may benefit from such long-acting treatment.

However, the potential long-term side effects with the first-generation depot antipsychotics, such as the development of depressive symptoms, have been reported to be a problem. ${ }^{7}$ Some second-generation antipsychotic depots are available, with risperidone, 
paliperidone, and olanzapine being marketed, and could be more beneficial than the first-generation antipsychotics in bipolar disorder maintenance treatment.

This review presents current data on the role of longacting second-generation antipsychotics, focusing on depot risperidone for maintenance treatment in patients with bipolar disorder. First, the efficacy of oral risperidone in bipolar disorder is reviewed, as well as the benefits of use of RLAI compared with the oral formulations. Second, the studies of RLAI in the treatment of bipolar patients are reviewed. In addition, the profiles of bipolar patients who could benefit more specifically from RLAI are discussed.

\section{Oral risperidone in bipolar disorder Second-generation antipsychotics and bipolar disorder}

In recent times, second-generation antipsychotics have demonstrated mood-stabilizing properties in patients with bipolar disorder. Evidence for the efficacy of second-generation antipsychotics is mainly focussed on the acute treatment of bipolar disorder. In acute mania, studies have demonstrated the efficacy of second-generation antipsychotics as monotherapy as well as in combination with mood stabilizers (lithium or valproate). Few studies of second-generation antipsychotic monotherapy have been conducted in bipolar depression. ${ }^{8}$ Olanzapine was associated with a modest antidepressant effect ${ }^{9}$ and quetiapine showed efficacy in bipolar depression. ${ }^{10}$

In this context, some psychiatrists have continued secondgeneration antipsychotic medication after remission of an acute mood episode, and therefore have suggested evidence of their efficacy as maintenance therapy. Currently, olanzapine, aripiprazole, and quetiapine monotherapy combined with lithium or valproate have received US Food and Drug Administration (FDA) approval for maintenance treatment in bipolar patients. ${ }^{8,11,12}$

\section{Mood-stabilizing properties of oral risperidone}

Data from randomized, controlled trials support the efficacy of risperidone monotherapy for the treatment of acute mania. Risperidone monotherapy was more effective than placebo and as effective as lithium or haloperidol in patients with acute mania. ${ }^{13-17}$ Since 2003, the FDA has approved the use of oral risperidone for the treatment of acute mania. The combination of lithium or valproate with risperidone has demonstrated superior efficacy compared with lithium or valproate alone. ${ }^{18,19}$
There is no robust evidence demonstrating the efficacy of risperidone monotherapy in bipolar depression. One study compared the effect of risperidone in combination with paroxetine, risperidone alone, and paroxetine alone, in patients with bipolar depression. ${ }^{20}$ The results showed no significant difference in improvement in depressive symptoms between the three groups. However, this study was small $(n=30)$ and the dose of paroxetine in the combination group was lower than that used in the monotherapy group.

Another randomized study, STEP-BD (Systematic Treatment Enhancement Program for Bipolar Disorder), assessed the effectiveness and safety of antidepressant with risperidone, lamotrigine, and inositol augmentation in treatment-resistant bipolar depression..$^{21}$ The recovery rate was lower with risperidone $(4.6 \%)$, whereas the recovery rates with lamotrigine and inositol were $23.8 \%$ and $17.4 \%$, respectively.

The recent guidelines for bipolar disorder proposed risperidone only in combination with antidepressants for bipolar depression to reduce the risk of switch to mania. ${ }^{22-24}$ Risperidone can be considered only as an antimanic agent.

The long-term efficacy of oral risperidone has mainly been assessed in preventing manic relapse. Initially, several studies examined the efficacy and safety of risperidone added on to mood stabilizers in the acute and continuation treatment of mania over a six-month period. ${ }^{25-28}$ The results indicated that the combination of risperidone with mood stabilizers was effective for acute and continuation treatment of mania. Furthermore, risperidone as add-on therapy did not induce depressive symptoms.

More recent studies have demonstrated the effectiveness, efficacy, and safety of oral risperidone monotherapy for acute and continuation treatment of mania. ${ }^{17,29}$ Risperidone did not induce depressive symptoms as part of combination therapy. A study of patients with bipolar II disorder has assessed the effectiveness and tolerability of oral risperidone monotherapy and in combination with mood stabilizers for acute and continuation treatment of hypomania. At the six-month follow-up, the results indicated that risperidone alone or in combination was effective. However, risperidone appeared to be more protective against hypomania than against depressive recurrences. ${ }^{26}$

\section{Risperidone long-acting injection: pharmacology, pharmacokinetics and benefits on treatment adherence}

Risperidone is the first second-generation antipsychotic for which a long-acting form has been marketed. With the 
difference of the delayed forms of first-generation antipsychotics, RLAI was developed in the form of microspheres containing the active ingredient, with progressive release by hydration. The product half-life in this long-acting form is assessed at three to six days, with elimination time after the first injection up to eight weeks. Delivery is done every 15 days by intramuscular injection, most often in the gluteus maximus muscle. Pharmacokinetic studies have demonstrated the existence of progressive slow release after the first injection, with a period of three weeks before obtaining the correct plasma level. The optimal plasma level is then obtained within four to six weeks, before progressively decreasing in two weeks. ${ }^{30}$ Continuing use of oral risperidone is therefore recommended for three weeks after the first injection.

The pharmacokinetic parameters of long-acting formulations have several advantages compared with oral forms. Firstly, the bioavailability is less random by avoiding gastrointestinal absorption and the effect of first-pass hepatic metabolism, obtaining greater concentrations at the level of the central nervous system. ${ }^{31}$ The doses administered by long-acting injections are therefore markedly lower than those used orally for the same effect and possibly improve tolerance to treatment. ${ }^{32}$

The ideal stability of the plasma level of the product compared with the oral form is also an important element. The use of long-acting risperidone ensures improved stability in product time and of its active metabolite (risperidone + 9-hydroxyrisperidone). ${ }^{33}$ The latter feature improves efficacy, by avoiding variations in plasma levels outside of the therapeutic zone.

Tolerance of long-acting risperidone has been widely studied. ${ }^{32,34,35}$ There does not seem to be a major difference in terms of neurologic (extrapyramidal) effects, endocrine (hyperprolactinemia) tolerance, and weight gain between the oral and long-acting forms. The adverse drug reactions linked to injection (pain, inflammation) seem less frequent with long-acting risperidone compared with first-generation antispychotics. ${ }^{34}$ It is also advisable to highlight the absence of overdose risk and administration being controlled, especially in the case of a suicidal medical history. ${ }^{32}$

Long-acting injectable formulations of second-generation antipsychotics could combine the effect of oral second-generation antipsychotics in patients with bipolar disorder and the benefits of depot formulation, with the assurance of steady medication delivery and improved adherence.

\section{Studies of long-acting risperidone injection in bipolar disorder Switching from an oral antipsychotic} As monotherapy

An open-label trial conducted during a 12-month follow-up period, studied the switch to depot risperidone in 10 patients in stable remission previously treated with low-dose oral second-generation antipsychotic monotherapy ${ }^{36}$ The results found no statistically or clinically significant change between the baseline and endpoint scores on the Young Mania Rating Scale (YMRS), Hamilton Rating Scale for Depression (HAM-D), and Brief Psychiatric Rating Scale (BPRS). The Clinical Global Improvement Scale (CGI-S) significantly decreased. Evaluation of patients' and caregivers' satisfaction using a 10-point visual analog scale indicated a high level of satisfaction at the endpoint. Concerning safety assessment, there was a significant improvement on the Extrapyramidal Symptom Rating Scale and a stable body weight after the switch from the oral antipsychotic to RLAI (Table 1).

A recent double-blind, 24-month randomized trial has assessed the usefulness of RLAI in monotherapy versus placebo to prevent mood episodes in patients with bipolar I disorder. ${ }^{37}$ Inclusion criteria were the existence of a current or recent acute manic or mixed episode (with YMRS score $\geq 20$ ), or the need for euthymic patients (CGI-S score $\leq 3$ ) to change treatment for safety reasons. Patients with more than four mood episodes per year during the last two years before the study were excluded. After an openlabel phase, patients who remained stable were randomized in two groups, ie, a placebo group and an RLAI continuation group. The results showed a significantly longer time to recurrence in the RLAI group $(P<0.001)$. Recurrence was noted in 45 patients (29\%) in the RLAI group versus 78 patients $(52 \%)$ in the placebo group. Concerning the type of mood episode, time to recurrence was significantly longer for elevated-mood episodes in the RLAI group $(P<0.001)$, while there was no significant difference for depressive episodes between the two groups $(P=0.805)$. Weight gain was more important in the RLAI group than in the placebo group. The incidence of extrapyramidal symptoms (EPS) was low, and serum prolactin concentrations increased from baseline during open-label RLAI stabilization and decreased from the double-blind baseline in both placebo and RLAI groups.

In combination with other mood stabilizers

In a retrospective observational study, ${ }^{38} 12$ manic or hypomanic patients, with a medical history of poor therapeutic 


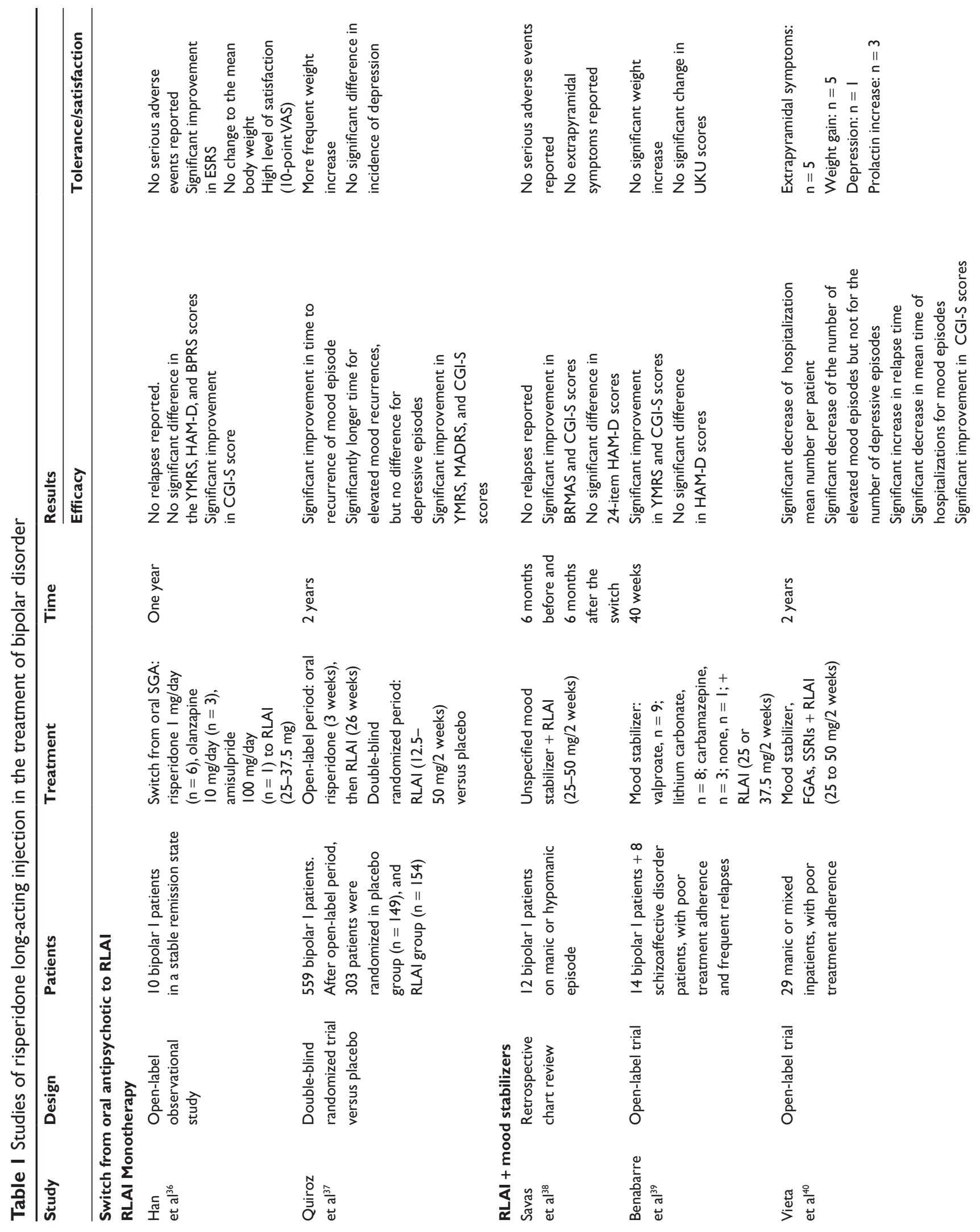



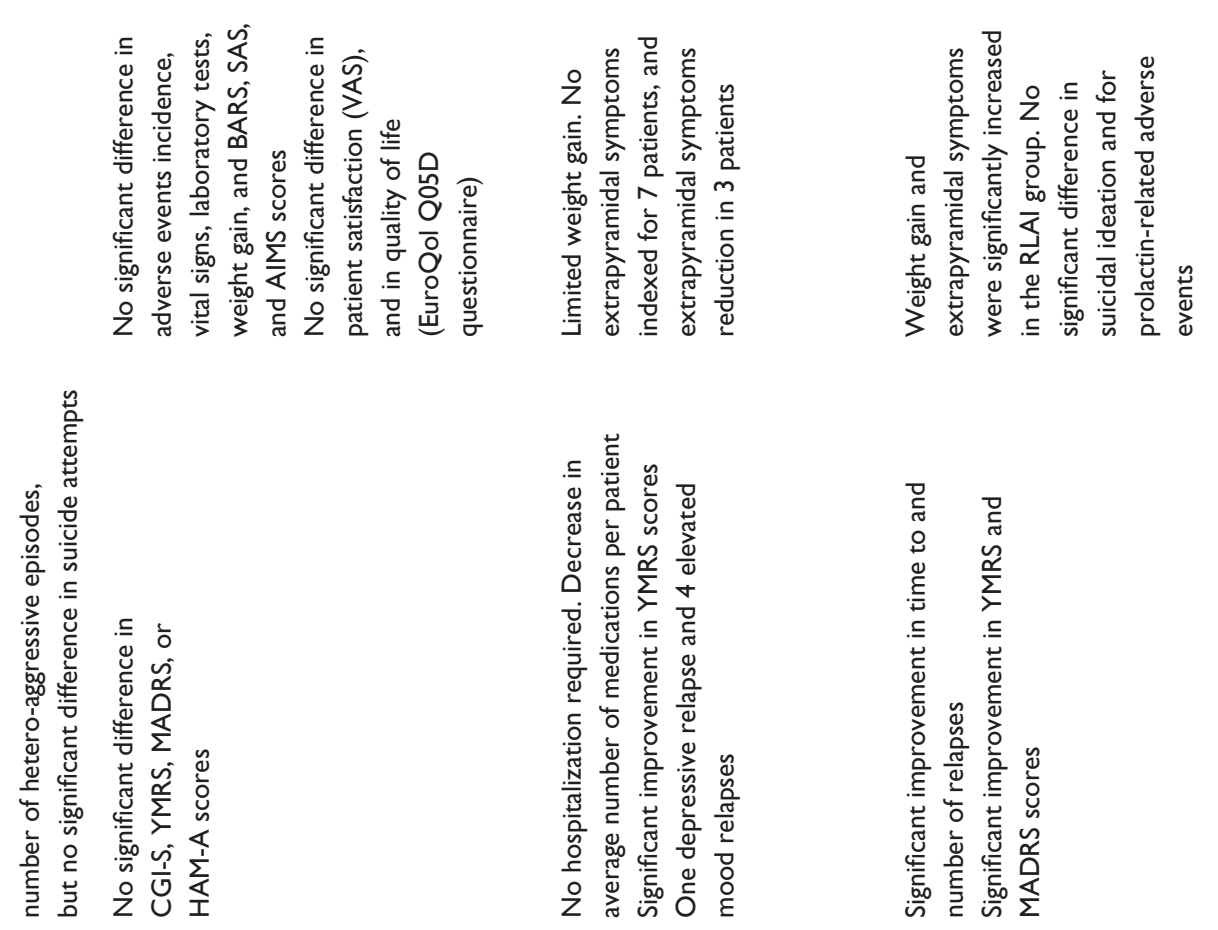

$\frac{n}{c}$
$\stackrel{+}{0}$
$\stackrel{0}{E}$
0

กั๊

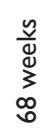

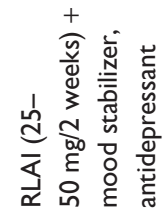
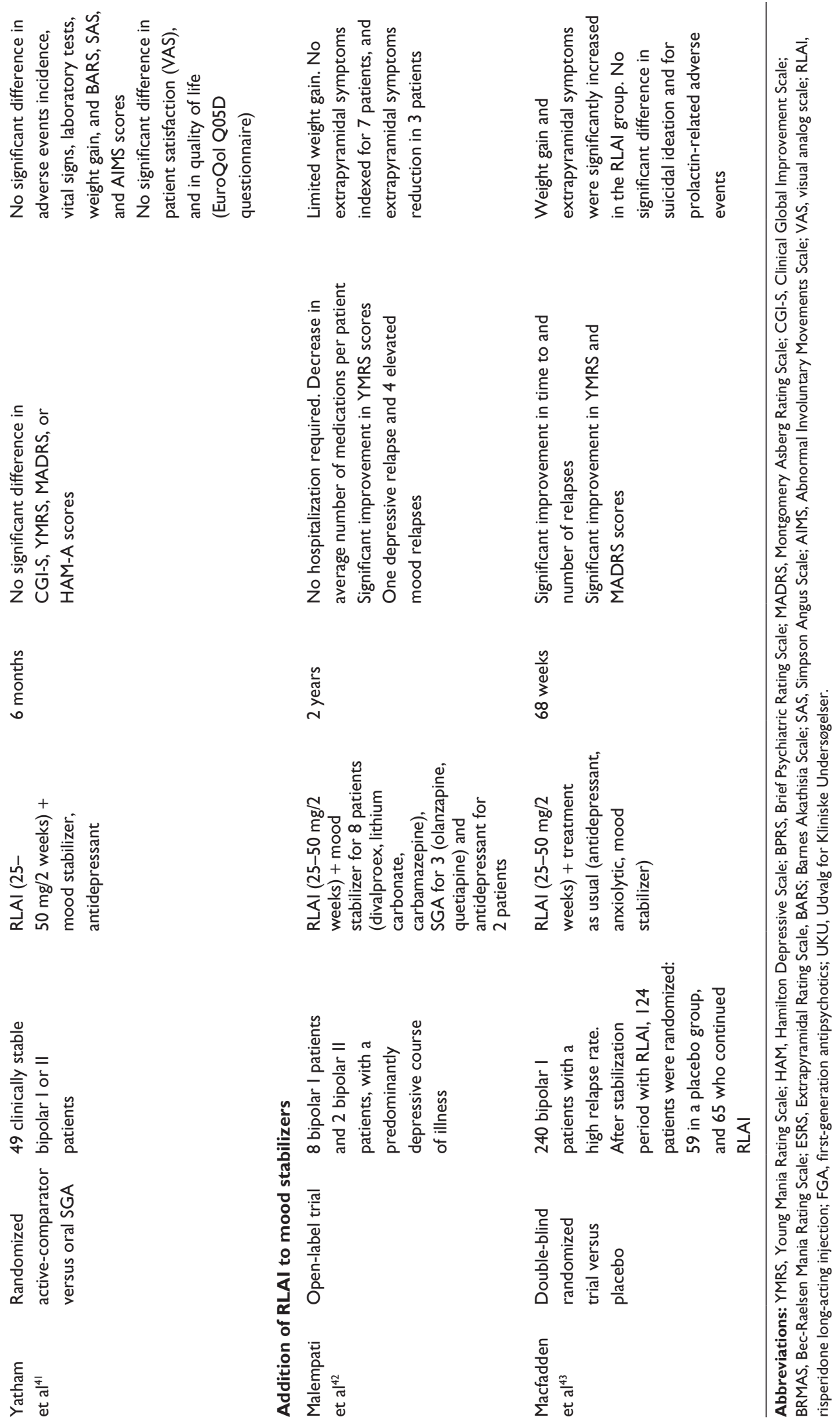
compliance, were assessed for six months after the switch to RLAI in combination with previous mood stabilizers. The results found early and long-lasting improvement on the Bech-Rafaelsen Mania Rating Scale (BRMAS) and of the CGI-S after treatment with RLAI. No significant modification of the 24-item HAM-D was observed, and no thymic episodes occurred after treatment with depot risperidone. The adverse drug reactions listed with RLAI were limited, with no EPS-related events documented.

Benabarre et al monitored 14 bipolar and eight schizoaffective patients over a period of 40 weeks. ${ }^{39}$ All patients presented with low therapeutic compliance caused by a high level of relapses. The oral antipsychotic treatments initially prescribed were replaced by RLAI and other current mood stabilizers were maintained. Under long-acting risperidone treatment, the authors observed significant improvements of YMRS and CGI-S scores $(P<0.001)$.

Another observational study, including 29 patients with an initial diagnosis of manic or mixed state, monitored over two years, assessed the replacement of an oral antipsychotic treatment by RLAI in combination with one or several other treatments (mood stabilizer, antipsychotic, antidepressant). ${ }^{40}$ The main objective was to compare the number of hospitalizations related to thymic relapses before and after the introduction of RLAI in patients identified as noncompliant or barely compliant. The number of hospitalizations turned out to be significantly lower during the period of RLAI $(P<0.006)$. The study demonstrated a significant reduction in the number of hospitalizations for manic or mixed decompensation under RLAI $(P<0.007)$. By contrast, no significant difference was demonstrated regarding hospitalizations for depressive episodes $(P=$ $0.73)$. An increase in time to relapse was also observed $(P$ $<0.001)$, along with a reduction in the average duration of hospitalizations $(P<0.001)$, after the patients were treated with RLAI.

An open randomized study compared RLAI with an oral antipsychotic in stable patients with Type I or II bipolar disorder. ${ }^{41}$ Once stabilized by the combination of a mood stabilizer and oral antipsychotic, with or without an antidepressant, 49 patients were randomized in two groups and monitored for a period of six months. In the first group $(n=23)$ the oral antipsychotic was replaced by RLAI; the other 26 patients continued their previous oral antipsychotic treatment. Efficacy assessments (CGI-S, YMRS, MADRS, and Hamilton Anxiety Rating Scale), as well as neurologic and biologic tolerance assessments, did not demonstrate a significant difference between the two groups.

\section{Adjunctive use with mood stabilizers}

An observational study assessed adjunctive use of RLAI over a period of two years in 10 bipolar patients with predominantly depressive polarity. ${ }^{42}$ RLAI was introduced either because of poor compliance with oral treatment or because of poor tolerance or lack of efficacy. Eight patients received one or more mood stabilizers in combination, three received another antipsychotic, and two an antidepressant. The addition of RLAI enabled improvement of YMRS scores, as well as a $50 \%$ reduction in the average number of coprescribed treatments. The authors found a minimum increase in MADRS between the start and end of the study, with occurrence of a single depressive decompensation with a favorable outcome under psychotherapy. One manic episode, one hypomanic episode, and two mixed episodes were described. An improvement in psychosocial functioning was also noted, with resumption of professional activity in five patients. The treatment was well tolerated in all patients. EPS were absent in seven patients and reduced in three patients, when compared with their prior medication therapy. The average weight gain of $2 \mathrm{~kg}$ over two years was considered moderate.

A randomized, double-blind study monitored a group of 240 patients with bipolar I disorder over a duration of 68 weeks, following thymic decompensation. ${ }^{43}$ The main characteristic of patients in this study was a high number of thymic episodes (at least four) over the previous 12 months. After 16 weeks receiving RLAI combined with one or more treatments (antidepressants, mood stabilizers, anxiolytics), 124 who patients stabilized for at least four weeks were randomized into two groups. Sixty-five patients therefore received RLAI as adjunctive therapy, and 59 received placebo. The addition of RLAI increased the time to relapse $(P=0.010)$ and decreased the number of relapses $(P=0.011)$ compared with the placebo group, regardless to the type of thymic episode. EPS and weight gain $(P=0.002)$ were found to have a significantly greater incidence in the RLAI group. Potential prolactin-related adverse effects (erectile dysfunction, decreased libido, amenorrhea) and mean changes in laboratory values for glucose, cholesterol, and triglycerides were similar between the adjunctive RLAI and placebo groups.

\section{Impact on therapeutic compliance}

Some authors describe fewer relapses when moving from an oral form of antipsychotic to RLAI, ${ }^{36,40}$ probably because the latter formulation enables better compliance. ${ }^{31}$ 
Several studies have tried to define the relevance of RLAI in the specific group of noncompliant or barely compliant patients. ${ }^{38-40}$ In specific category of patients, the efficacy results were in favor of the long-acting formulation. Moreover, these studies highlight good tolerance of the product in the long-acting form, suggesting better adherence to treatment. Vieta et $\mathrm{a}^{40}$ have demonstrated a significant improvement in overall compliance, including with oral treatments, and a decrease in the rate of poor compliance from $86 \%$ to $27.5 \%$ with RLAI $(P<0.0001)$.

\section{Discussion Profile of eligible patients}

The bulk of the data suggests that monotherapy RLAI is effective in the preventive treatment of manic but not depressive recurrences in bipolar patients. The profile of patients who can benefit from the use of RLAI seems to be influenced by both patient- and illness-related factors.

\section{Patient-related factors}

Patient compliance after prescription of prophylactic treatment is an individual factor which plays a central role in the prevention of thymic recurrences. In practice, we can describe a subgroup of bipolar patients with a low level of treatment compliance. This subgroup shows an improvement in compliance after introducing RLAI (as monotherapy or as part of a combination). Studies including patients with a history of poor adherence to treatment have found that RLAI improved therapeutic efficacy in the long term, with an improvement in the YMRS and CGI-S scores, increased time before relapse, and a reduction in the number of hospitalizations associated with the same (or even better) level of treatment tolerance.

The subpopulation of nonadherent bipolar patients is associated with particular sociodemographic and clinical characteristics, such as young age, male gender, mainly single or separated marital status, low educational levels, psychiatric comorbidity, drug abuse, and personality disorders. ${ }^{40,44}$ These characteristics could be an argument for the priority use of RLAI in long-term treatment. However, full compliance with treatment is difficult to obtain in the majority of chronic illnesses. ${ }^{45}$ The use of RLAI in maintenance treatment for all bipolar patients is therefore relevant. Nevertheless, the long-term gain obtained after the introduction of RLAI will be greater in patients with poor adherence to treatment.

\section{Bipolar disorder-related factors Dominant thymic polarity}

The studies presented in this review indicate that RLAI is effective in preventing relapse in mania during long-term treatment. More specifically, the studies indicate efficacy as monotherapy and in combination with mood stabilizers in preventing relapse of manic episodes. Efficacy in preventing relapse of depressive episodes was also shown but only in treatments combining RLAI with other mood stabilizers. No data were available to assess efficacy as monotherapy in preventing relapse of depression.

The RLAI monotherapy studies are the most commonly provided in monitoring manic or mixed episodes, where the latest episode is often correlated to the thymic polarity of the relapse. ${ }^{46}$ These patients were more at risk of manic recurrences which could explain a lower rate of depressive relapse than manic relapses. However, compared with firstgeneration antipsychotics, monotherapy with RLAI does not worsen symptoms of depression in patients presenting mania or mixed states.

Since the early 1980s, several studies have assessed the efficacy and safety of first-generation depot antipsychotics in patients with bipolar disorder. A review of these studies showed that these agents decrease the frequency and severity of manic symptoms during maintenance treatment, but also that they may worsen the depressive phase of bipolar disorder. ${ }^{7}$ Some studies reported several suicides as a result of worsening depression. ${ }^{47}$

The first-generation antipsychotics do not show any relevance in the maintenance treatment of bipolar patients in contrast with RLAI which, through the absence of induction of depressive symptoms, may be considered as a prophylactic treatment in monotherapy in patients with manic polarity.

The absence of data in bipolar patients with depressive polarity does not seem to allow the long-term use of RLAI in monotherapy, but it can be used in combination with other mood stabilizers.

\section{Severity of bipolar disorder}

To our knowledge, there is no consensual definition of severe bipolar disorder. The severity of the disorder can be defined using a dimensional or longitudinal approach, as well as categoric criteria. The current classifications tend to assess severity of illness on a dimensional basis according to intensity of symptoms (number of symptoms present), their impact, or presence of psychotic symptoms. Occurrence of hyperthymic episodes, using precise categories, such as mixed episode, defines the severity of bipolar disorder. From a longitudinal viewpoint, chronicity, number of recurrences, adherence to treatment, and impact on quality of life can be taken into account to define the severity of bipolar disorder. 
The studies shown in this review most often assessed severity using dimensional scales (eg, YMRS, MADRS, and CGI-S) to assess changes during treatment. Some authors have also assessed the use of RLAI in patient categories associated with severe bipolar disorder, ie, patients with frequent recurrences (at least four times over the last 12 months), ${ }^{43}$ ie, patients who presented poor adherence to treatment. ${ }^{38-40}$ The use of RLAI combined with other mood stabilizers in the treatment of severe bipolar disorder appeared to be more effective than combinations of oral medication, and remain associated with maintenance of good tolerance. ${ }^{38-40,43}$

Use of oral second-generation antipsychotics in bipolar disorder is recommended during thymic episodes with psychotic symptoms. It will be noticed that no study has attempted to demonstrate the efficacy of RLAI in patients presenting during relapses of thymic episodes associated with psychotic symptoms by taking into account the presence of these symptoms as well. The relevance of RLAI for this subgroup of patients still needs to be demonstrated.

\section{Clinical limitations}

The studies described in this review demonstrate a safety profile for RLAI consistent with that previously observed in short- and long-term studies, with no emergence of new safety issues in patients with schizophrenia. ${ }^{34,48-50}$ When compared with oral formulations, the long-acting formulation seems to cause fewer neurologic adverse effects, especially EPS, while prolactin-related adverse events occur at a similar rate.

Among the second-generation antipsychotics, risperidone appears to be the molecule with the higher risk for neurologic and endocrine adverse events. ${ }^{51}$ However, such reactions remain less frequent in comparison with those associated with the first-generation antipsychotics. ${ }^{52}$ When using risperidone, weight gain and metabolism disorders occur as frequently as for most second-generation antipsychotics. ${ }^{51}$

At present, there are no specific studies dealing with the tolerance of RLAI in comparison with other mood stabilizers, such as lithium and the anticonvulsants. Tolerance profiles of these last medications are associated with another range of adverse effects, such as thyroid abnormalities and renal or hepatic reactions, which differ from those described with RLAI use. Consequently, the benefit:risk ratio for RLAI, as for other medications, should be evaluated individually, taking into account personal and familial antecedents and results of clinical examination or biologic assessments. RLAI may not be suitable for all patients with bipolar disorder.

\section{Methodological limitations}

Most of the studies included in this review suffered from several methodologic limitations. Only two large studies were randomized controlled trials, ${ }^{37,43}$ and most were small, open-label trials or retrospective reviews. Some studies also included a mixture of bipolar and schizoaffective patients or used combination medications, and none compared RLAI with oral risperidone or oral second-generation antipsychotics. Given the limited evidence, other long-term studies employing randomized, controlled and blinded designs, along with dominant thymic polarity patients (especially depressive polarity) are needed.

\section{Positioning of RLAI in guidelines for bipolar disorder management}

Most guidelines recommended use of long-term medication according to the dominant thymic polarity. ${ }^{22,23}$ First-line medications are lithium, valproate, aripiprazole, and olanzapine for predominantly manic patients and quetiapine and lamotrigine for predominantly depressive patients.

Because of the low level of evidence for RLAI, it is mentioned in guidelines as second- or third-line prophylactic treatment for bipolar disorder (except for the CANMAT guidelines for bipolar disorder ${ }^{24}$ which recommend RLAI as a first-line therapy). However, few guidelines have considered the usefulness of RLAI versus other maintenance treatment options in poorly compliant bipolar patients at high risk for manic relapse or as adjunctive medication with another mood stabilizer. ${ }^{22,23}$

\section{Conclusion}

If use of long-acting first-generation antipsychotics is to be avoided in the treatment of bipolar patients due to increased risk of promoting appearance of depressive symptoms, and in particular increasing the risk of suicide, long-acting secondgeneration antipsychotics, such as RLAI, may be considered for maintenance treatment of bipolar patients. RLAI appears to be particularly suitable for patients presenting with poor adherence or with severe bipolar disorder. However, the present data necessitate taking into account the individual safety profile and the thymic polarity of the bipolar patient. RLAI can be used as monotherapy in patients with manic polarity, but RLAI should always be considered for use in combination with at least one other mood stabilizer in patients with depressive polarity.

In clinical practice, the prescription of RLAI in psychotic patients is still not very high by psychiatrists $(<10 \%) .{ }^{53}$ The negative attitude of patients to RLAI seems to be correlated 
with a low level of information from psychiatrists about the medication, ${ }^{54}$ because patients who receive treatment with RLAI report an increase in satisfaction with their treatment. ${ }^{55}$

The clinical use of long-acting second-generation antipsychotics in bipolar patients requires that better information on the formulation be relayed from psychiatrists to their patients.

\section{Disclosure}

The authors report no conflicts of interest in this work.

\section{References}

1. Fajutrao L, Locklear J, Priaulx J, et al. A systematic review of the evidence of the burden of bipolar disorder in Europe. Clin Pract Epidemiol Ment Health. 2009;23;5(1):3.

2. Sanchez-Moreno J, Martinez-Aran A, Tabarés-Seisdedos R, et al. Functioning and disability in bipolar disorder: An extensive review. Psychother Psychosom. 2009;78:285-297.

3. Kilbane EJ, Gokbayrak NS, Galynker I, et al. A review of panic and suicide in bipolar disorder: Does comorbidity increase risk? J Affect Disord. 2009;115(1-2):1-10.

4. Lingam R, Scott J. Treatment non-adherence in affective disorders. Acta Psychiatr Scand. 2002;105(3):164-172.

5. Scott J, Pope M. Self-reported adherence to treatment with mood stabilizers, plasma levels, and psychiatric hospitalization. Am J Psychiatry. 2002;159(11):1927-1929.

6. Kane JM. Utilization of long-acting antipsychotic medication in patient care. CNS Spectr. 2006;11(12 Suppl 14):1-7; quiz 7-8.

7. Bond DJ, Pratoomsri W, Yatham LN. Depot antipsychotic medications in bipolar disorder: A review of the literature. Acta Psychiatr Scand Suppl. 2007;(434):3-16.

8. Yatham, LN. Atypical antipsychotics for bipolar disorder. Psychiatr Clin North Am. 2005;28(2):325-347.

9. Tohen M, Vieta E, Calabrese J, et al. Efficacy of olanzapine and olanzapine-fluoxetine combination in the treatment of bipolar I depression. Arch Gen Psychiatry. 2003;60(11):1079-1088.

10. Weisler RH, Calabrese JR, Thase ME, et al. Efficacy of quetiapine monotherapy for the treatment of depressive episodes in bipolar I disorder: A post hoc analysis of combined results from 2 doubleblind, randomized, placebo-controlled studies. J Clin Psychiatry. 2008;69(5):769-782.

11. Keck PE Jr, Calabrese JR, McIntyre RS, et al. Aripiprazole monotherapy for maintenance therapy in bipolar I disorder: A 100-week, double-blind study versus placebo. J Clin Psychiatry. 2007;68(10):1480-1491.

12. Suppes T, Vieta E, Liu S, et al. Maintenance treatment for patients with bipolar I disorder: Results from a North American study of quetiapine in combination with lithium or divalproex (trial 127). Am J Psychiatry. 2009;166(4):476-488.

13. Hirschfeld RM, Keck PE Jr, Kramer M, et al. Rapid antimanic effect of risperidone monotherapy: A 3-week multicenter, double-blind, placebo-controlled trial. Am J Psychiatry. 2004;161(6):1057-1065.

14. Khanna S, Vieta E, Lyons B, et al. Risperidone in the treatment of acute mania: Double-blind, placebo-controlled study. Br J Psychiatry. 2005;187:229-234.

15. Rendell JM, Geddes JR. Risperidone in long-term treatment for bipolar disorder. Cochrane Database Syst Rev. 2006(4):CD004999.

16. Segal J, Berk M, Brook S, et al. Risperidone compared with both lithium and haloperidol in mania: A double-blind randomized controlled trial. Clin Neuropharmacol. 1998;21(3):176-180.
17. Smulevich AB, Khanna S, Eerdekens M, et al. Acute and continuation risperidone monotherapy in bipolar mania: A 3-week placebo-controlled trial followed by a 9-week double-blind trial of risperidone and haloperidol. Eur Neuropsychopharmacol. 2005;15(1):75-84.

18. Sachs GS, Grossman F, Ghaemi SN, et al. Combination of a mood stabilizer with risperidone or haloperidol for treatment of acute mania: A double-blind, placebo-controlled comparison of efficacy and safety. Am J Psychiatry. 2002;159(7):1146-1154.

19. Yatham LN, Grossman F, Augustyns I, et al. Mood stabilisers plus risperidone or placebo in the treatment of acute mania. International, doubleblind, randomised controlled trial. Br J Psychiatry. 2003;182:141-147.

20. Shelton RC, Stahl SM. Risperidone and paroxetine given singly and in combination for bipolar depression. J Clin Psychiatry. 2004;65(12):1715-1719.

21. Nierenberg AA, Ostacher MJ, Calabrese JR, et al. Treatment-resistant bipolar depression: A STEP-BD equipoise randomized effectiveness trial of antidepressant augmentation with lamotrigine, inositol, or risperidone. Am J Psychiatry. 2006;163(2):210-216.

22. Goodwin GM. Evidence-based guidelines for treating bipolar disorder: Revised second edition - recommendations from the British Association for Psychopharmacology. J Psychopharmacol. 2009;23(4):346-388.

23. Malhi GS, Adams D, Lampe L, et al. Clinical practice recommendations for bipolar disorder. Acta Psychiatr Scand Suppl. 2009;(439):27-46.

24. Yatham LN, Kennedy SH, Schaffer A, et al. Canadian Network for Mood and Anxiety Treatments (CANMAT) and International Society for Bipolar Disorders (ISBD) collaborative update of CANMAT guidelines for the management of patients with bipolar disorder: Update 2009. Bipolar Disord. 2009;11(3):225-255.

25. Bowden CL, Myers JE, Grossman F, et al. Risperidone in combination with mood stabilizers: A 10-week continuation phase study in bipolar I disorder. J Clin Psychiatry. 2004;65(5):707-714.

26. Vieta E, Gasto C, Colom F, et al. Role of risperidone in bipolar II: An open 6-month study. J Affect Disord. 2001;67(1-3):213-219.

27. Yatham LN, Binder C, Kusumakar V, et al. Risperidone plus lithium versus risperidone plus valproate in acute and continuation treatment of mania. Int Clin Psychopharmacol. 2004;19(2):103-109.

28. Yatham LN, Binder C, Riccardelli R, et al. Risperidone in acute and continuation treatment of mania. Int Clin Psychopharmacol. 2003;18(4):227-235.

29. Vieta E, Brugue E, Goikolea JM, et al. Acute and continuation risperidone monotherapy in mania. Hum Psychopharmacol. 2004; 19(1):41-45.

30. Knox ED, Stimmel GL. Clinical review of a long-acting, injectable formulation of risperidone. Clin Ther. 2004;26(12):1994-2002.

31. McEvoy JP. Risks versus benefits of different types of long-acting injectable antipsychotics. J Clin Psychiatry. 2006;67:Suppl 5:15-18.

32. Rainer, MK. Risperidone long-acting injection: A review of its long term safety and efficacy. Neuropsychiatr Dis Treat. 2008;4(5):919-927.

33. Nesvag R, Hendset M, Refsum H, et al. Serum concentrations of risperidone and 9-OH risperidone following intramuscular injection of long-acting risperidone compared with oral risperidone medication. Acta Psychiatr Scand. 2006;114(1):21-26.

34. Kane JM, Eerkedens M, Lindenmayer JP, et al. Long-acting injectable risperidone: Efficacy and safety of the first long-acting atypical antipsychotic. Am J Psychiatry. 2003;160(6):1125-1132.

35. Keith $\mathrm{S}$. Use of long-acting risperidone in psychiatric disorders: Focus on efficacy, safety and cost-effectiveness. Expert Rev Neurother. 2009;9(1):9-31.

36. Han C, Lee MS, Pae CU, et al. Usefulness of long-acting injectable risperidone during 12-month maintenance therapy of bipolar disorder. Prog Neuropsychopharmacol Biol Psychiatry. 2007;31(6):1219-1223.

37. Quiroz JA, et al. Risperidone long-acting injectable monotherapy in the maintenance treatment of bipolar I disorder. Biol Psychiatry. 2010 Mar 12. [Epub ahead of print].

38. Savas HA, Yumru M, Ozen ME, et al. Use of long-acting risperidone in the treatment of bipolar patients. J Clin Psychopharmacol. 2006;26(5):530-531. 
39. Benabarre A, Castro P, Sanchez-Moreno J, et al. [Efficacy and safety of long-acting injectable risperidone in maintenance phase of bipolar and schizoaffective disorder]. Actas Esp Psiquiatr. 2009;37(3):143-147. Spanish.

40. Vieta E, Nieto E, Autet A, et al. A long-term prospective study on the outcome of bipolar patients treated with long-acting injectable risperidone. World J Biol Psychiatry. 2008;9(3):219-224.

41. Yatham LN, Fallu A, Binder CE. A 6-month randomized open-label comparison of continuation of oral atypical antipsychotic therapy or switch to long acting injectable risperidone in patients with bipolar disorder. Acta Psychiatr Scand Suppl. 2007;(434):50-56.

42. Malempati RN, Bond DJ, Yatham LN, et al. Depot risperidone in the outpatient management of bipolar disorder: A 2-year study of 10 patients. Int Clin Psychopharmacol. 2008;23(2):88-94.

43. Macfadden W, Alphs L, Haskins JT, et al. A randomized, double-blind, placebo-controlled study of maintenance treatment with adjunctive risperidone long-acting therapy in patients with bipolar I disorder who relapse frequently. Bipolar Disord. 2009;11(8):827-839.

44. El-Mallakh RS. Medication adherence and the use of long-acting antipsychotics in bipolar disorder. J Psychiatr Pract. 2007;13(2):79-85.

45. Llorca PM. Partial compliance in schizophrenia and the impact on patient outcomes. Psychiatry Res. 2008;161(2):235-247.

46. Calabrese JR, Vieta E, El-Mallakh R, et al. Mood state at study entry as predictor of the polarity of relapse in bipolar disorder. Biol Psychiatry. 2004;56(12):957-963.

47. Littlejohn R, Leslie F, Cookson J. Depot antipsychotics in the prophylaxis of bipolar affective disorder. Br J Psychiatry. 1994;165(6):827-829.
48. Simpson GM, Mahmoud RA, Lasser RA, et al. A 1-year double-blind study of 2 doses of long-acting risperidone in stable patients with schizophrenia or schizoaffective disorder. J Clin Psychiatry. 2006;67(8): 1194-1203.

49. Fleischhacker WW, Eerdekens M, Karcher K, et al. Treatment of schizophrenia with long-acting injectable risperidone: A 12-month open-label trial of the first long-acting second-generation antipsychotic. J Clin Psychiatry. 2003;64(10):1250-1257.

50. Chue P, Eerdekens M, Augustyns I, et al. Comparative efficacy and safety of long-acting risperidone and risperidone oral tablets. Eur Neuropsychopharmacol. 2005;15(1):111-117.

51. Leucht S, Komossa K, Rummel-Kluge C, et al. A meta-analysis of head-to-head comparisons of second-generation antipsychotics in the treatment of schizophrenia. Am J Psychiatry. 2009;166(2):152-163.

52. Leucht S, Corves C, Arbter D, et al. Second generation versus firstgeneration antipsychotic drugs for schizophrenia: A meta-analysis. Lancet. 2009;373(9657):31-41.

53. Hamann J, Mendel R, Heres S, et al. How much more effective do depot antipsychotics have to be compared to oral antipsychotics before they are prescribed? Eur Neuropsychopharmacol. 2010;20(4):276-279.

54. Jaeger M, Rossler W. Attitudes towards long-acting depot antipsychotics: A survey of patients, relatives and psychiatrists. Psychiatry Res. 2010;175(1-2):58-62.

55. Lindenmayer JP, Jarboe K, Bossie CA, et al. Minimal injection site pain and high patient satisfaction during treatment with long-acting risperidone. Int Clin Psychopharmacol. 2005;20(4):213-221.
Patient Preference and Adherence

\section{Publish your work in this journal}

Patient Preference and Adherence is an international, peer-reviewed, open access journal focusing on the growing importance of patient preference and adherence throughout the therapeutic continuum. Patient satisfaction, acceptability, quality of life, compliance, persistence and their role in developing new therapeutic modalities and compounds to

\section{Dovepress}

optimize clinical outcomes for existing disease states are major areas of interest. This journal has been accepted for indexing on PubMed Central. The manuscript management system is completely online and includes a very quick and fair peer-review system. Visit http://www.dovepress.com/ testimonials.php to read real quotes from published authors. 